\title{
Moving horizon estimation for continuous glucose monitoring
}

\author{
Theresa Kruse $^{1}$, Knut Graichen ${ }^{2}$ \\ ${ }^{1}$ Institute of Measurement, Control and Microtechnology, Ulm University \\ 89081 Ulm, Germany \\ ${ }^{2}$ Friedrich-Alexander University Erlangen-Nürnberg, Chair of Automatic Control \\ 91058 Erlangen, Germany \\ theresa.kruse@uni-ulm.de, knut.graichen@fau.de
}

\begin{abstract}
Continuous glucose monitoring (CGM) systems have great advantages for the treatment of diabetes. Common commercial sensors are placed in the subcutaneous tissue and continuously measure (1-5 $\mathrm{min}$ ) a signal correlated to the glucose concentration in the interstitial fluid (ISF). As the glucose concentration in the ISF and the blood compartment differ, especially after meal or insulin injection, blood glucose (BG) estimation methods improve the consistency of CGM sensor glucose values and standard blood glucose measurements. However, reference measurements (selfmonitoring blood glucose measurements) are typically required for the calibration of CGM sensors. Thus, the sensor calibration also depends on the quality of BG estimation. In this paper we present a BG estimation method based on moving horizon estimation combined with an adaptive noise variance estimation. Compared with the common estimation methods, Kalman filtering and signal smoothing, the presented method achieves an improve of BG estimation. In addition, the method leads to an improved estimation of past BG values, leading to better calibration results.
\end{abstract}

Keywords: continuous glucose monitoring, blood glucose estimation, moving horizon estimation, noise adaptation

\section{Introduction}

Continuous glucose monitoring (CGM) is a benefit for all diabetes patients. Instead of using self monitoring blood glucose (SMBG) devices to control the blood glucose (BG) level multiple times a day, it allows to observe the BG the whole day, even during the night when normally no measurements are done. Another advantage is to set hypo- and hyperglycamic alarms if the measured or the predicted BG concentration reaches a critical threshold. These benefits can help to control BG better and to avoid health critical situations [1, 2, 3]. Moreover a continuous and robust determination of BG is the premise for a closed-loop blood glucose control. Currently there are two different commercial CGM systems available, an electrochemical one based on enzymatic oxidation and an optical system using a glucosedependent fluorescence technique. All systems have in common, that they measure glucose concentration in the interstitial fluid (IG). It is well known, that the IG and BG concentration differ especially after disorders like eating or insulin administration [4]. This effect is shown in Fig.

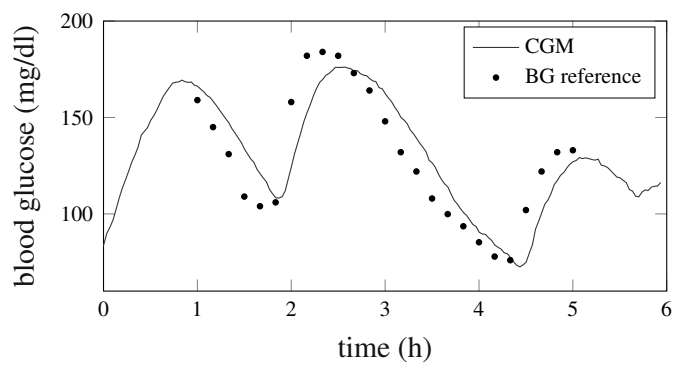

Fig. 1: Smoothed CGM signal and BG measurements 1 which shows an IG signal measured with a CGM sensor and the corresponding reference BG measurements. It can be attributed to the diffusion process between the two compartments, leading to a lag time and a damped ISF glucose concentration [5].

ICBES 119-1 
The IG concentration and the sensor signal $y=h(I G, p)$ are in general linked by an output function $h$. As the sensor parameters $p$ may vary between different sensors and/ or drift during sensor lifetime, it is neccessary to estimate the parameters once or twice a day. Different estimation methods have been presented in the past $[6,7]$. Almost all of them depend on SMBG measurements as reference data. However, the afore mentioned differences between the BG and IG concentrations have a significant influence on the sensor calibration and typically lead to an incorrect parameter identification [8]. Different methods for compensating the diffusion effect in the measuring signal were presented in the past, typically they are based on filtering methods [9], such as Kalman filtering [10]. Most filters are based on a constant model and cannot be adapted to changes in the noise variance. The effect of the diffusion process, however, is sometimes neglected and the CGM signal is simply smoothed instead [11].

In contrast to this, we present a BG estimation method based on moving horizon estimation (MHE) with an adaptive noise estimation. In the following a brief introduction of the estimation method is given and the state transition and sensor model is described. Based on this model, an adaptation procedure for the process and measurement noise is presented. Finally, the procedure is tested on real data and compared with other estimation methods. Moreover the influence of the estimation accuracy on the calibration process is analyzed. MHE provides not only a current estimate but also a smoothed past signal, which can be used for calibration and, due to a smaller estimation error, it leads to a better identification of the sensor parameters.

\section{Methods}

\subsection{Moving horizon estimation}

Moving horizon estimation is a state estimation method minimizing a cost function defined on a sliding window of length $N[12,13]$. Based on a discrete-time system with

$$
\begin{array}{r}
x_{k}=f\left(x_{k-1}, w_{k-1}\right) \\
y_{k}=h\left(x_{k}, v_{k}\right),
\end{array}
$$

where $x_{k}$ is the state variable vector and $y_{k}$ is the measurement vector, the cost function includes the weighted norm of measurement $v_{k}$ and process noise $w_{k}$ of the past horizon $N$ at time $k$. Thus, the optimization problem defining MHE is of the following form

$$
\min _{\{x\}_{j \mid k}^{k \mid k}} \sum_{j=k-N+1}^{k} \frac{1}{\sigma_{v}}\left\|v_{j \mid k}\right\|^{2}+\frac{1}{\sigma_{w}}\left\|w_{j-1 \mid k}\right\|^{2},
$$

where $\{x\}_{j \mid k}^{k \mid k}$ are the estimated states $x_{k-N+1}, . ., x_{k}$ at time $k$ and $\sigma_{w}^{2}=\operatorname{var}(w)$ and $\sigma_{v}^{2}=\operatorname{var}(v)$ are the variances of the process noise, respectively, measurement noise, equivalent to minimizing uncertainty. Compared with Kalman filtering, the noise must be uncorrelated and zero-mean, but not necessarily Gaussian distributed.

\subsection{State transition and sensor model}

Referring to the two-compartment model introduced by Rebrin et al. [5], the BG-to-IG dynamic can be described by

$$
\frac{\mathrm{d} i(t)}{\mathrm{d} t}=\frac{1}{\tau}(b(t)-i(t)),
$$

where $i(t)$ and $b(t)$ are the IG, respectively, BG signal and $\tau$ is the time constant of the diffusion process. Assuming that the sensor output function is of the form $y(t)=p_{0} i(t)+p_{1}$ an uncalibrated BG signal $x^{b}=p_{0} b(t)+p_{1}$ and an uncalibrated IG signal $x^{i}=p_{0} i(t)+p_{1}$ can be introduced. Provided that the sensor parameters $p_{0}$ and $p_{1}$ change slowly, (3) is also valid for the uncalibrated signals [14]

$$
\frac{\mathrm{d} x^{i}(t)}{\mathrm{d} t}=p_{0} \frac{\mathrm{d} i(t)}{\mathrm{d} t}=\frac{1}{\tau}\left(x^{b}(t)-x^{i}(t)\right)
$$


Discretization of (4) with the time step size $\Delta t$ and modeling the BG dynamic $x^{b}$ with an autoregressive (AR) model leads to the following discrete state-space representation

$$
\begin{aligned}
x_{k+1}^{b} & =2 x_{k}^{b}-x_{k-1}^{b}+w_{k} \\
x_{k+1}^{i} & =x_{k}^{i}+\frac{\Delta t}{\tau}\left(x_{k}^{b}-x_{k}^{i}\right) \\
y_{k} & =x_{k}^{i}+v_{k} .
\end{aligned}
$$

Once the ideal, uncalibrated BG value $x_{k}^{b}$ is estimated, the $\mathrm{BG}$ concentration is obtained from $b_{k}=\frac{y_{k}^{b}-p_{1}}{p_{0}}$.

\subsection{Blood glucose estimation}

The optimization variables of the MHE formulation (2) for the given problem are the uncalibrated, noise-free BG values $x_{k-N+1 \mid k}^{b}, \ldots, x_{k \mid k}^{b}$. In the following, a matrix notation is used with $N$-dimensional vectors consisting of the past $k-N-1, . ., k$ variables. Thus, the process noise $w_{k}=\left(w_{k-N \mid k}, \ldots, w_{k-1 \mid k}\right)^{T}$ and the measurement noise $v_{k}=\left(v_{k-N+1 \mid k}, \ldots, v_{k \mid k}\right)^{T}$ are defined as

$$
w_{k}=C x_{k}^{b}+D z_{k} \quad \text { and } \quad v_{k}=y_{k}-x_{k}^{i}
$$

with the IG signal $x_{k}^{i}$

$$
x_{k}^{i}=A x_{k}^{b}+B z_{k},
$$

the initial states $z_{k}:=\left(x_{k-N}^{i}, x_{k-N-1}^{b}, x_{k-N}^{b}\right)^{T}$ and the matrices

$$
A=\frac{\Delta t}{\tau}\left(\begin{array}{ccccc}
0 & 0 & 0 & \cdots & 0 \\
1 & 0 & 0 & \cdots & 0 \\
a & 1 & 0 & \cdots & 0 \\
\vdots & \ddots & \ddots & \ddots & \vdots \\
a^{N-2} & \cdots & a & 1 & 0
\end{array}\right), B=\left(\begin{array}{ccc}
a & 0 & \frac{\Delta t}{\tau} a^{0} \\
\vdots & \vdots & \vdots \\
a^{N} & 0 & \frac{\Delta t}{\tau} a^{N-1}
\end{array}\right), C=\left(\begin{array}{ccccc}
1 & 0 & 0 & \cdots & 0 \\
-2 & 1 & 0 & \cdots & 0 \\
1 & -2 & 1 & \cdots & 0 \\
\vdots & \ddots & \ddots & \ddots & \vdots \\
0 & \cdots & 1 & -2 & 1
\end{array}\right), D=\left(\begin{array}{ccc}
0 & 1 & -2 \\
0 & 0 & 1 \\
\vdots & \ddots & \vdots \\
0 & \cdots & 0
\end{array}\right)
$$

with $a=1-\frac{\Delta t}{\tau}$. With respect to these notations, the MHE formulation (2) can be transformed to

$$
\min _{x_{k}^{b}}\left(\left\|w_{k}\right\|_{Q_{k}^{-1}}^{2}+\left\|v_{k}\right\|_{R_{k}^{-1}}^{2}\right)
$$

where the weighting matrices $Q_{k}=\operatorname{cov}\left(w_{k}\right)$ and $R_{k}=\operatorname{cov}\left(v_{k}\right)$ correspond to the covariance matrices of process and measurement noise. Substituting (6) into the optimization problem (9) leads to a quadratic programming problem

$$
\min _{x_{k}^{b}}\left\{x_{k}^{b^{T}}\left(A^{T} R^{-1} A+C^{T} Q^{-1} C\right) x_{k}^{b}-\left(\left(y_{k}-B z_{k}\right)^{T} R^{-1} A-z_{k}^{T} D^{T} Q^{-1} C\right) x_{k}^{b}\right\}
$$

which can be solved by matrix inversion

$$
\hat{x}_{k}^{b}=\left(A^{T} R^{-1} A+C^{T} Q^{-1} C\right)^{-1}\left(A^{T} R^{-1}\left(y_{k}-B z_{k}\right)-C^{T} Q^{-1} D z_{k}\right)=H y_{k}+G z_{k}
$$

or efficient linear solvers.

The initial states of vector $z_{k}$ are set to the corresponding solution of the previous estimation steps $k-1$ and $k-2$, i.e.

$$
z_{k}=\left(x_{k-N}^{i}, x_{k-N-1}^{b}, x_{k-N}^{b}\right)^{T}=\left(\hat{x}_{k-N \mid k-1}^{i}, \hat{x}_{k-N-1 \mid k-2}^{b}, \hat{x}_{k-N \mid k-1}^{b}\right)^{T} .
$$


In the initialization step $(k=N)$, the initial states has to be estimated as well. Therefore, the optimization vector is extended with the initial values $x_{0}=\left(z_{k}, x_{k}^{b}\right)$ and the optimization problem can be modified with $A_{0}=[B A]$ and $C_{0}=[D C]$ and replacing $B_{0}$ and $D_{0}$ with zero matrices.

In contrast to other estimation methods, MHE estimates an actual value $\hat{x}_{k \mid k}^{b}$ as well as past values $\hat{x}_{j \mid k}^{b}$ $(j>k-N+1)$. These lead to a smoothed BG and IG signal

$$
\hat{X}_{k-N+1, \ldots, k}^{b}=\hat{x}_{k}^{b} \quad \text { and } \quad \hat{X}_{k-N+1, \ldots, k}^{i}=\hat{x}_{k}^{i}=A \hat{x}_{k}^{b}+B z_{k} .
$$

Note that $\hat{X}_{j}^{b}$ for $j \leq k-N+1$ is based on the estimates $\hat{x}_{k-N+1 \mid k}^{b}$.

\subsection{Noise adaptation}

Optimal performance of the MHE is only guaranteed by knowing the variance of measurement $\sigma_{v, k}^{2}$ and process noise $\sigma_{w, k}^{2}$. In general, these two parameters are unknown and have to be estimated. Furthermore, both parameters may vary over the measurement time. Hence, the adaptation of the variances has a direct impact on the estimation quality [15]. An example for over- and underestimation of noise is presented in Fig. 2. Underestimation of measurement noise leads to a very noisy signal, whereas overestimation of measurement noise, respectively, underestimation of process noise results in a time delayed estimate.

In the following, a method is presented which is based on the notion of equivalent degrees of freedom [16]. It allows to predict measurement and process variance and achieves a simple adaptation of the variances. To this end, we have a closer look at the measurement error $v$ and the process error $w$. Assuming that the process $w_{j-1 \mid k}$ and measurement noise $v_{j \mid k}$ of a horizon with

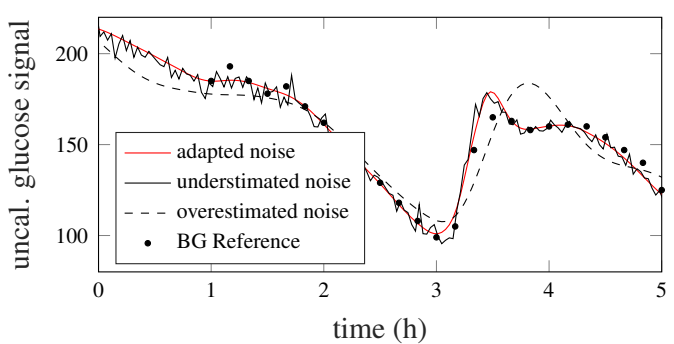

Fig. 2: Estimated BG signal with noise adaption (solid line), underestimated noise (dashed line) and overestimated noise (dotted line) length $n$ and $j=k-n+1, \ldots k$ are part of a distribution with variance $\sigma_{w, k}$, respectively $\sigma_{v, k}$, the covariance matrices $R_{k}$ and $Q_{k}$ correspond to $R_{k}=\sigma_{v, k}^{2} I$ and $Q_{k}=\sigma_{w, k}^{2} I$. With the quotient $\gamma_{k}=\frac{\sigma_{v, k}^{2}}{\sigma_{w, k}^{2}}$ of the variances of process and measurement noise the measurement noise is given by

$$
v_{k}=\left(I-A H\left(\gamma_{k}\right)\right) y_{k}-(A G+B) z_{k} .
$$

With a sufficiently large horizon length $(N \Delta t \approx 2 \tau)$, the smoothed estimation results $x_{k-N+1 \mid k}^{b}$ and $x_{k-N+1 \mid k}^{i}$ change only minimally due to an extension of the horizon $(N=N+m$ and $k=k+m)$. If the variance of these starting points is neglected compared to the measurement and process variance, the covariance matrix has the following form

$$
\operatorname{cov}\left(v_{k}\right)=\left(I-A H\left(\gamma_{k}\right)\right) \operatorname{cov}\left(y_{k}\right)\left(I-A H\left(\gamma_{k}\right)\right)^{T} .
$$

Furthermore, the covariance of the uncalibrated, noise-free BG signal $\operatorname{cov}\left(x_{k}^{b}\right)=\sigma_{w}^{2} C^{-1}\left(C^{-1}\right)^{T}$ and the covariance of the ideal, uncalibrated IG signal $\operatorname{cov}\left(x_{k}^{i}\right)=\sigma_{w}^{2} A C^{-1}\left(C^{-1}\right)^{T} A^{T}$ only depends on the variance matrix of the process noise. Moreover, since the covariance matrix of the measurement vector is composed of the covariance matrix of the measurement noise and of the uncalibrated, noise-free IG signal, we have

$$
\operatorname{cov}\left(y_{k}\right)=\sigma_{v}^{2} I+\sigma_{w}^{2} A\left(C^{-1}\right)^{T} C^{-1} A .
$$

Due to insertion of (16) in (15) and applying the matrix inversion lemma, the covariance matrix of the measurement and process noise is given by:

$$
\operatorname{cov}\left(v_{k}\right)=\sigma_{v}^{2}\left(I-A H\left(\gamma_{k}\right)\right)
$$


With the expected value of the sum of squared measurement errors $S S V_{k}=v_{k}^{T} v_{k}$ which can be transformed to $E\left(S S V_{k}\right)=E\left(v_{k}^{T} v_{k}\right)=E\left(\operatorname{tr}\left(v_{k} v_{k}^{T}\right)\right)=E\left(\operatorname{tr}\left(\operatorname{cov}\left(v_{k}\right)\right)\right)$ and the corresponding variance, the measurement noise becomes $\sigma_{v, k}^{2}=\frac{E\left(S S V_{k}\right)}{n-s\left(\gamma_{k}\right)}$, where $s\left(\gamma_{k}\right)=\operatorname{tr}\left(A H\left(\gamma_{k}\right)\right)$. This results in a consistent estimate of the measurement noise variance

$$
\hat{\sigma}_{v, k}^{2}=\frac{S S V_{k}}{n-s\left(\gamma_{k}\right)} .
$$

An estiamtor for the process error variance can be derived in a similar manner, starting with the covariance of process noise and sum of squared process errors $S S W_{k}=w_{k}^{T} w_{k}$, i.e.

$$
\hat{\sigma}_{w, k}^{2}=\frac{S S W_{k}}{S\left(\gamma_{k}\right)} .
$$

Initial values for the variances should be set to typical values, which are then adapted. Moreover, the noise adaptation horizon $n$ should be chosen considerably greater than $N$, since a long estimation horizon $N$ creates high computational costs with only little to no improvement in accuracy, while the estimation accuracy of the variances strongly correlates with the number of data points. The $S S V$ and $S S W$ are therefore calculated from the smoothed signal

$$
S S W_{k}=\sum_{j=k-n+1}^{k} \hat{X}_{j}^{b}-2 \hat{X}_{j-1}^{b}+\hat{X}_{j-2}^{b} \quad \text { and } \quad S S V_{k}=\sum_{j=k-n+1}^{k} \hat{X}_{j}^{i}-y_{j} .
$$

The adaptation process can be repeated after $n$ steps.

\section{Results}

The presented method is tested on real CGM signals that were measured with the Fibersense CGM system [17]. The system is based on glucose depending fluorescence measurements and has a sampling rate of one measurement in two minutes $(\Delta t=2 \mathrm{~min})$. The estimation based on MHE is compared to two other estimation methods, Kalman filtering (KF) and CGM signal smoothing using a moving average filter (MA). In addition, the effect of $\mathrm{BG}$ estimation on the parameter identification is analyzed.

The data set corresponds to 8 type 1 and 8 type 2 diabetic patients. The sensor was worn for 28 days. At four clinical visits (day 1, 7, 15, 28) reference data was collected every 10 minutes over a period of four hours using the Yellow Springs Instrument (YSI) 2300 STAT Plus glucose analyzer (YSI Life Sciences, Yellow Springs, $\mathrm{OH}$ ). The MHE horizon for estimation and noise adaptation are set to $N=10$ and $n=50$, respectively. The Kalman filter is based on the same state-space representation (5) as MHE with average values for process and measurement error variances. A time constant of $\tau=6 \mathrm{~min}$ for the diffusion process is used for both methods.

\subsection{Blood glucose estimation}

The filtered CGM signals $\hat{x}_{k}^{b}$ generated with MHE as well as KF and MA are compared based on their consistency with the measured reference data during the clinical monitoring. Note that only the actual BG signal $\hat{x}_{k \mid k}^{b}$ is used for the evaluation of the MHE results. The sensor parameters are identified by least square estimation for each clinical visit and each estimation method.

The performance of the estimation methods is evaluated based on three metrics; the mean absolute relative difference (MARD), the root mean square error (RMSE) and the maximal relative absolute difference (max RAD) of the four clinical measurements of all 16 patients. Table 1 lists median and lower $\left(\mathrm{Q}_{1}\right)$ and upper quartiles $\left(\mathrm{Q}_{3}\right)$ of these metrics. The MHE signal leads to the best results for all of the three metrics, followed by the KF signal. The MA signal represents a filtered IG signal that does not account for the diffusion process between IG and BG and leads to the worst results. KF and MHE differ mainly in the adaptation of the process and measurement noise. This is very important for the sensor used here, because 
it is not worn for 7-10 days as usual with electrochemical sensors but for 4 weeks and the parameters can change significantly over this time.

Table 1: Blood glucose estimation results (median $\left[\mathrm{Q}_{1}, \mathrm{Q}_{3}\right]$ ) of the three different estimation methods.

\begin{tabular}{lrrr}
\hline method & MARD $(\%)$ & RMSE $(\mathrm{mg} / \mathrm{dl})$ & maxRAD $(\mathrm{mg} / \mathrm{dl})$ \\
\hline MHE & $6.1[4.3,9.3]$ & $8.2[5.7,11.2]$ & $19.0[13.0,29.5]$ \\
KF & $7.1[4.6,9.6]$ & $9.6[6.9,12.5]$ & $20.0[14.9,31.2]$ \\
MA & $7.8[5.3,11.6]$ & $11.6[8.1,15.2]$ & $22.8[14.3,31.9]$ \\
\hline
\end{tabular}

\subsection{Calibration error}

In the normal online application, the actual BG estimate is of utmost importance. However, the smoothed estimation results $\hat{X}_{k-N+1}^{b}=\hat{x}_{k-N+1 \mid k}^{b}$ can be used for the calibration procedure. All three metrics of this signal (pMHE) are even significantly smaller in comparison to the results of the MHE signal based on the actual estimates $\hat{x}_{k \mid k}^{b}$ (median MARD $=5.1 \%$, median $\mathrm{RMSE}=7.1 \mathrm{mg} / \mathrm{dl}$ and median maxRAD $=14.2 \mathrm{mg} / \mathrm{dl}$ ). The improvement is due to additional smoothing of the signal and better compensation of the time delay. Changes in blood glucose only lead to changes in the ISF glucose concentration after a certain time, so estimation methods such as MHE and KF can only react to these changes at a later stage. Since calibration of CGM systems is typically based on blood glucose reference measurements, the accuracy of blood glucose estimation has significant influence on the precision of sensor parameter estimation.

Different methods for sensor calibration were presented in the past $[6,7]$. In the following, it is referred to the simplest one, the two-point calibration. Two reference measurements $\left(b_{1}, b_{2}\right)$ and the time corresponding BG estimation results $\left(\hat{x}_{1}^{b}, \hat{x}_{2}^{b}\right)$ are used to calculate the sensor parameters $p_{0}=b_{2}-b_{1} / \hat{x}_{2}^{b}-\hat{x}_{1}^{b}$ and $p_{1}=b_{1}-p_{0} \hat{x}_{1}^{b}$. As the
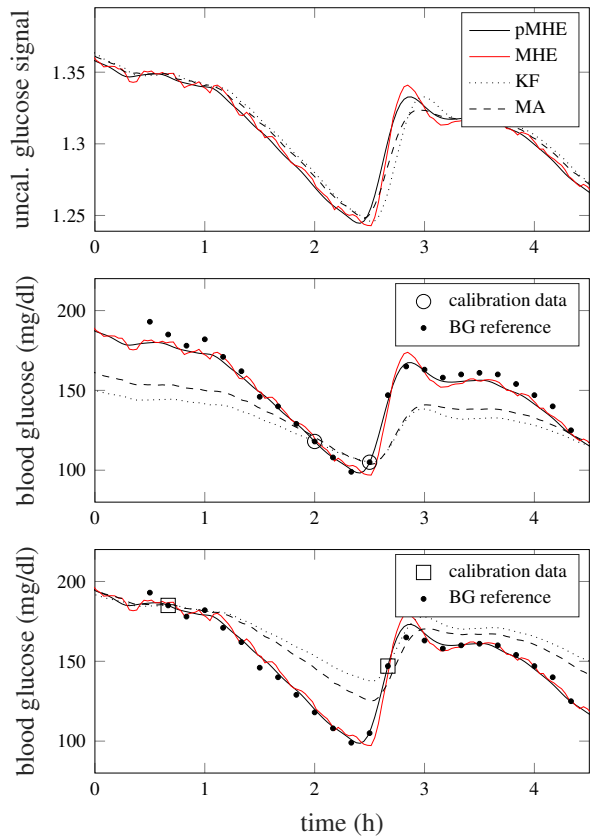

Fig. 3: Uncalibrated BG signals of a CGM signal calculated with different estimation methods (upper figure) and calibrated signals for two different two-point calibrations (circled and squared BG references). sampling time of the CGM system is rather large, interpolation between two time steps is often necessary.

A two-point calibration data set is generated for each clinical session, consisting of each possible combination of two reference measurements. For each reference combination and for each estimated uncalibrated BG signal (MHE, pMHE, KF and MA), the sensor parameters are identified and the blood glucose concentration is calculated. The metrics MARD and RMSE are estimated for each calibration scenario. Note that they correspond to the estimation and the calibration error. Fig. 3 shows an example for two different calibration scenarios. The upper figure shows the uncalibrated BG signals. The BG profiles shown in the middle and lower figure are generated by two-point calibration with the marked reference data (circle, square). Although the estimates of the uncalibrated blood glucose signal vary only slightly, the effects on the calibrated signal are clear visible.

Table 2 lists median and quartiles of MARD and RMSE of the clinical visits of all patients for all possible calibrations. The method based on the pMHE signal has the smallest median and interquartile distance of 

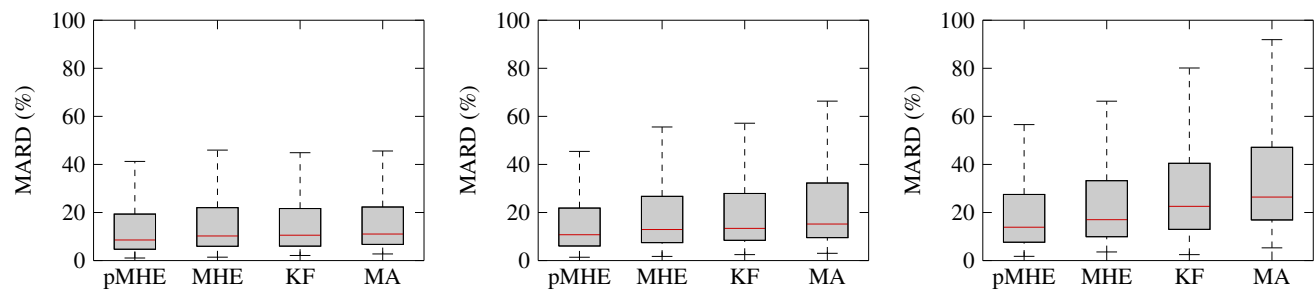

Fig. 4: Boxplot of MARD for calibrations with calibration data of a region with almost no dynamical behaviour $\frac{|\Delta B G|}{\Delta t}<1 \mathrm{mg} / \mathrm{dl}$ (left), with at least one reference BG measurement with medium rate of change $\frac{|\Delta B G|}{\Delta t}<3 \mathrm{mg} / \mathrm{dl}$ (middle) and with at least one of a high dynamic range $\frac{|\Delta B G|}{\Delta t}>3 \mathrm{mg} / \mathrm{dl}$ (right).

MARD and RMSE. Even more significant are the results using reference data with high dynamical behaviour in BG. The BG rate of change from a previous to an actual glucose concentration serves as a measure for the BG dynamic. The calibration set is divided into three groups; the first group contains of reference data of regions with almost stationary behaviour $(|\Delta B G| / \Delta t<1 \mathrm{mg} / \mathrm{dl})$, the second group with at least one reference data point with medium rate of change $(1 \mathrm{mg} / \mathrm{dl}<|\Delta B G| / \Delta t<3 \mathrm{mg} / \mathrm{dl})$, and the third group consists of all calibration combinations having at least one calibration point in a high dynamic range $(|\Delta B G| / \Delta t>3 \mathrm{mg} / \mathrm{dl})$. The median and quartiles of MARD and RMSE are shown in Fig.4. Besides the BG estimation error, further errors, e. g. sensor errors or an error of the reference measurement, lead to a poor identification of the sensor parameters. Hence, high MARD and RMSE are produced. For reasons of clarity, these outliers are not shown in the figure.

The results of calibrations with at least one reference data point with a high rate of change are summarized in Tab. 3. The small estimation error of pMHE leads to a more accurate identification of the sensor parameters and therefore to smaller MARD and RMSE.

Table 2: Blood glucose estimation results (median $\left.\left[\mathrm{Q}_{1}, \mathrm{Q}_{3}\right]\right)$ of all possible two-point calibrations.

\begin{tabular}{lrr}
\hline method & MARD $(\%)$ & RMSE $(\mathrm{mg} / \mathrm{dl})$ \\
\hline pMHE & $10.1[5.5,21.5]$ & $18.2[10.5,37.2]$ \\
MHE & $12.0[6.8,25.6]$ & $21.5[12.7,43.2]$ \\
KF & $12.6[7.4,26.7]$ & $23.0[14.1,45.0]$ \\
MA & $14.3[8.3,29.3]$ & $26.0[16.5,50.0]$ \\
\hline
\end{tabular}

Table 3: Blood glucose estimation results (median $\left[\mathrm{Q}_{1}, \mathrm{Q}_{3}\right]$ ) of all possible two-point calibrations with at least one calibration measurement with high dynamic range.

\begin{tabular}{lrr}
\hline method & MARD $(\%)$ & RMSE $(\mathrm{mg} / \mathrm{dl})$ \\
\hline pMHE & $13.9[7.6,27.5]$ & $24.8[15.2,42.6]$ \\
MHE & $17.0[9.9,33.2]$ & $29.4[18.4,53.8]$ \\
KF & $22.6[13.0,40.5]$ & $36.7[22.8,74.2]$ \\
MA & $26.4[16.9,47.1]$ & $42.8[27.6,86.2]$ \\
\hline
\end{tabular}

\section{Conclusion}

The presented BG estimation method combines moving horizon estimation with noise adaptation. It is shown that an improved real-time BG estimation is achieved in contrast to MA or Kalman filtering. The improvement is achieved by compensating the time delay and by optimizing the estimator using noise adaptation. The adaptation of measurement and process noise is of advantage if the parameters change due to the aging of the sensor. This is especially important for the sensor data used here, as the wearing time of the sensor is significantly longer compared to conventional systems.

The method also has the advantage that in addition to the current estimated value a past blood glucose signal is simultaneously estimated. This signal has a significantly lower noise and shows better compensation of the time delay. This advantage is particularly noticeable when calibrating the sensor with reference BG measurements. Since an accurate estimate of the blood sugar leads to a better identification of the sensor parameters and these have a critical role in the accuracy of the blood glucose estimation. 


\section{References}

[1] D. Deiss, J. Bolinder, J. P. Riveline, T. Battelino, E. Bosi, N. Tubiana-Rufi, D. Kerr, and M. Phillip, "Improved glycemic control in poorly controlled patients with type 1 diabetes using real-time continuous glucose monitoring," Diabetes Care, vol. 29, no. 12, pp. 2730-32, 2006.

[2] S. Garg, H. Zisser, S. Schwartz, T. Bailey, R. Kaplan, S. Ellis, and L. Jovanovic, "Improvement in glycemic excursions with a transcutaneous, real-time continuous glucose sensor: A randomized controlled trial," Diabetes Care, vol. 29, no. 1, pp. 44-50, 2006.

[3] J.-C. Preiser, O. Lheureux, A. Thooft, S. Brimioulle, J. Goldstein, and J.-L. Vincent, "Near-Continuous Glucose Monitoring Makes Glycemic Control Safer in ICU Patients." Crit Care Med, vol. 46, no. 8, pp. 1224-29, aug 2018.

[4] A. Basu, S. Dube, M. Slama, I. Errazuriz, J. C. Amezcua, Y. C. Kudva, T. Peyser, R. E. Carter, C. Cobelli, and R. Basu, "Time lag of glucose from intravascular to interstitial compartment in humans." Diabetes Care, vol. 62, no. 12, pp. 4083-7, dec 2013.

[5] K. Rebrin, G. M. Steil, W. P. van Antwerp, and J. J. Mastrototaro, "Subcutaneous glucose predicts plasma glucose independent of insulin: implications for continuous monitoring," Am J Physiol, vol. 277, no. 3, pp. E561-E571, sep 1999.

[6] G. Acciaroli, M. Vettoretti, A. Facchinetti, and G. Sparacino, "Calibration of minimally invasive continuous glucose monitoring sensors: State-of-the-art and current perspectives," Biosensors, vol. 8, no. 1, pp. 1-17, 2018.

[7] D. Rodbard, "Continuous Glucose Monitoring: A Review of Successes, Challenges, and Opportunities," Diabetes Technol Ther, vol. 18, no. S2, pp. S2-3-S2-13, 2016.

[8] S. Guerra, A. Facchinetti, G. Sparacino, G. D. Nicolao, and C. Cobelli, "Enhancing the accuracy of subcutaneous glucose sensors: A real-time deconvolution-based approach," IEEE Trans. Biomed. Eng., vol. 59, no. 6, pp. 1658-1669, 2012.

[9] K. Rebrin and G. M. Steil, "Use of Subcutaneous Interstitial Fluid Glucose to Estimate Blood Glucose: Revisiting Delay and Sensor Offset," J Diabetes Sci Technol, vol. 4, no. 5, pp. 1087-1098, 2010.

[10] E. J. Knobbe and B. Buckingham, "The Extended Kalman Filter for Continuous Glucose Monitoring," Diabetes Technol Ther, vol. 7, no. 1, pp. 15-27, feb 2005.

[11] B. W. Bequette, "Continuous glucose monitoring: Real-time algorithms for calibration, filtering, and alarms," J Diabetes Sci Technol, vol. 4, no. 2, pp. 404-418, 2010.

[12] P. K. Findeisen, "Moving horizon state estimation of discrete time systems," Ph.D. dissertation, University of Wisconsin-Madison, 1997.

[13] J. B. Rawlings, "Moving Horizon Estimation," in Encyclopedia of Systems and Control. London: Springer, 2014, pp. 1-7.

[14] M. Vettoretti, A. Facchinetti, S. Del Favero, G. Sparacino, and C. Cobelli, "Online calibration of glucose sensors from the measured current by a time-varying calibration function and Bayesian priors," IEEE Trans. Biomed. Eng., vol. 63, no. 8, pp. 1631-41, 2016.

[15] A. Facchinetti, G. Sparacino, and C. Cobelli, "Online Denoising Method to Handle Intraindividual Variability of Signal-to-Noise Ratio in Continuous Glucose Monitoring," IEEE Trans. Biomed. Eng., vol. 58, no. 9, pp. 2664-71, sep 2011.

[16] G. Wahba, "Bayesian Confidence Intervals for the Cross-Validated Smoothing Spline," J R Stat Soc, vol. 45, no. 1, pp. 133-150, sep 1983.

[17] F. Küster, K. S. Nikolaus, A. J. Müller, R. Krivánek, C. Hasslacher, and M. Knuth, "First Clinical Evaluation of a New Percutaneous Optical Fiber Glucose Sensor for Continuous Glucose Monitoring in Diabetes," J Diabetes Sci Technol, vol. 7, no. 1, pp. 13-23, 2014. 